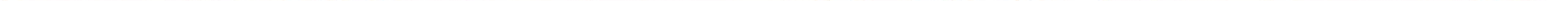




\section{El aporte del sector oficial a las expresiones de cultura y a la salvaguardia del patrimonio cul- tural, a veintidós años de la fir- ma de la paz. La cultura como un motor de desarrollo integral Max Araujo}

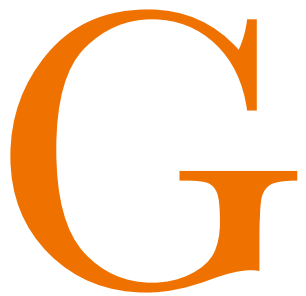

uatemala tiene, como todos los países y regiones, un movimiento cultural cotidiano, que se manifiesta de muchas formas: en expresiones culturales tradicionales, conocimientos ancestrales y en expresiones artísticas, tanto en centros urbanos como en sus áreas rurales, aunque hay muchas personas que lo niegan, porque lo ignoran o por los conceptos que tienen sobre cultura y su patrimonio. Confunden por ejemplo, cultura con arte, o con la educación escolar que se recibe por estudios formales. Una de las principales críticas es que el gobierno no apoya, o apoya poco, a pesar de la obligación que tiene establecida en su Constitución Política de la República, en la normativa legal que se ha emitido y en las convenciones e instrumentos internacionales que se han ratificado, que como consecuencia son de obligado cumplimiento. Entre los reclamos más fuertes está el que no se reconocen los derechos culturales de los pueblos indígenas, es decir de los herederos de las poblaciones que existían cuando se dio la conquista española en el siglo dieciséis. Una de las principales causas, se dice, es que cuando se obtuvo la independencia de España, en 1821, se construyó un Estado neocolonial y monocultural, en el que una cultura hegemónica, la de los ladinos o mestizos, que sustituyó a los criollos, descendientes directos de los peninsulares (de España), se impone sobre las culturas subordinadas, las de los indígenas. Otra causa, para el caso específico de las expresiones artísticas, se dice que estas, salvo excepciones reconocidas, no alcanzan los niveles de calidad que se tienen en otros países, por lo que la responsabilidad la tiene el gobierno porque no genera las condiciones apropiadas, ni de educación, ni para los apoyos que son necesarios. A pesar de esas críticas se reconoce y se valora el talento de artistas reconocidos, nacional e internacionalmente, que han surgido a lo largo de nuestra historia, incluyendo a algunos que han residido o residen en el extranjero. Lo más contemporáneo de las artes es conocido y desarrollado. Tendencias, estilos, escuelas, movimientos, cultores, que se dan en otras partes del mundo conviven con expresiones vernáculas. Las explicaciones, interpretaciones y los estudios abundan. Es por ello, porque he estado ligado al Ministerio de Cultura y Deportes desde hace más de veinte años, y porque milito en el mundo cultural de la ciudad de Guatemala, el principal centro urbano, que escribo este texto en el que presento un breve panorama de la situación actual de la obligación que tiene el sector gubernamental en la salvaguardia de las expresiones de cultura, 
entre las que se encuentran las tradicionales y ancestrales, un patrimonio cultural material, representado por inmuebles y muebles de los tres grandes periodos de su historia: prehispánico, colonial y republicano, así como los derechos culturales de los pueblos indígenas y las artísticas en general.

El país sufrió un conflicto armado que duró treinta y seis años. Se puso fin al mismo el 29 de diciembre de 1996, con la firma de la paz, entre el Estado y la guerrilla, previo firma de varios acuerdos para distintas áreas y sectores, que son un proyecto de nación, para lograr que sea más justa e incluyente, que no se han desarrollado como se esperaba, pero que han dado algunos resultados positivos, entre ellos los del área de cultura, aunque es mucho lo que falta por hacer, no solo porque las causas estructurales que dieron origen al conflicto armado se mantienen, sino porque también las sociedades son dinámicas, y porque hay circunstancias internas y externas que influyen, entre ellas las demandas y reconocimientos a los requerimientos de las distintas colectividades, especialmente las marginadas, derivadas de compromisos internacionales por normativas que se han suscrito. Para el caso de Guatemala se destacan los legítimos reclamos de los pueblos indígenas y de otros sectores vulnerables en cuanto al reconocimiento de sus derechos culturales, económicos, políticos y sociales. En el tema de derechos culturales de los pueblos indígenas se han dado avances importantes por la creación de instituciones y dependencias, por la emisión de leyes nacionales y por la ratificación de instrumentos internacionales relacionados con dichos derechos. Esos avances no son suficientes por los rezagos históricos que se arrastran desde hace más de cinco siglos, tres de la colonia y dos como república independiente. Es importante señalar que uno de los acuerdos previos a la firma de la paz es el de Identidad y Derecho de los Pueblos Indígenas, aunque ya habían sido reconocidos en la Constitución Política de la República, emitida en 1985. Sin embargo, no se trata de hacer una defensa oficiosa de las autoridades y de las instituciones de gobierno, sobre todo del Ministerio de Cultura y Deportes, al que se le asigna, cada año, un presupuesto deficitario para todo lo que debe hacer.

Para el panorama que hacemos en este trabajo uno de los grandes logros que se han conseguido es el haber llegado al convencimiento, entre los sectores afines, que la cultura debe ser un motor del desarrollo integral de Guatemala, es por ello que sus políticas culturales tienen ese concepto como un eje transversal a las mismas, siendo la política rectora «Culturas nacionales y desarrollo integral». A los valores inherentes de la cultura como identidad, cohesión social, autoestima, orgullo y sentido de pertenencia, se asume que también es economía.

Según un estudio realizado por Ernesto Piedras, mexicano, patrocinado por el BID, la cultura de Guatemala, representada por la diversidad de expresiones culturales, materiales e inmateriales, le aportan a su PIB un porcentaje de un 7.2 por ciento. Sin embargo a esta estimación se le debe agregar lo que aportan las actividades y empresas de la economía informal o sombra, ligada a expresiones culturales tradicionales, fiestas patronales, ciclos culturales como la cuaresma y la navidad o de sus artesanías, entre otros, más lo que genera el llamado turismo cultural con sus empresas conexas de transporte, alojamiento, alimentación y de diversión.

Para entender la parte de gobierno relacionado con cultura y su patrimonio, es necesario que se conozca cómo se integra el Sistema Nacional de Cultura, que existe de hecho, aunque no tiene una normativa legal que lo haya creado como tal, pero que funciona, con sus carencias y limitaciones, que comprende instituciones de gobierno, entidades no gubernamentales y personas de la sociedad civil, así como programas, proyectos y acciones, que tienen un sustento en la legislación cultural, que para el caso de Guatemala es rica y variada, como se demuestra con las publicaciones que sobre ese tema he realizado. Este sistema, según mi criterio, está integrado con el sector de gobierno y con el sector no gubernamental, en la siguiente forma: De la parte gubernamental. 1-De las entidades creadas específicamente para la cultura. En esta área se incluye a las entidades de gobierno que pertenecen a la estructura del Organismo Ejecutivo, pero también algunas que corresponden a los Organismos Legislativo y Judicial.

La primera parte de este sector comprende a las dependencias creadas específicamente para atender aspectos de cultura, entre los cuales se encuentran el Ministerio de Cultura y Deportes como ente rector, el Aporte para la Descentralización Cultural ADESCA, la Academia de Lenguas Mayas, el Consejo de Protección de la Antigua Guatemala, el Consejo Nacional del Libro CONALIBRO, entre otras. Dentro de esta área se incluye también al Ministerio de Educación Pública, a la Universidad de San Carlos de Guatemala, que se dedican a la educación, siendo que las segundas y la tercera entidad goza de un régimen jurídico especial por la autonomía que tiene, consagrada en la misma Constitución de la República. La educación es fundamental como un componente de la cultura, aunque en Guatemala se le separa de ese sector por aspectos administrativos y presupuestarios.

Es importante mencionar que los Ministerios de Cultura y Deportes y de Educación Pública, la Universidad de 


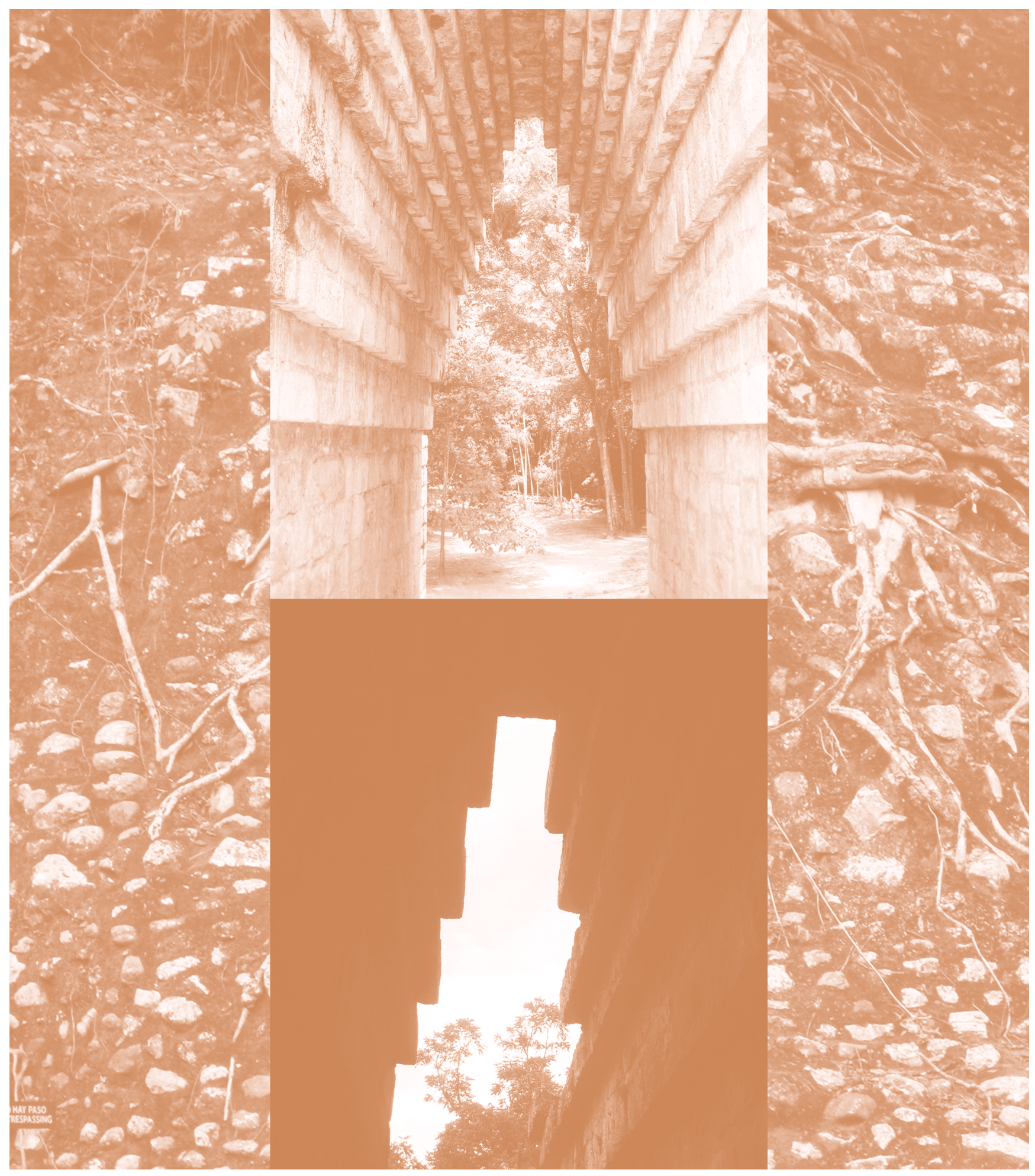

255 
San Carlos, y las otras instituciones mencionadas, tienen una estructura administrativa que comprende dependencias dedicadas a aspectos propios del sector cultura, por lo que el presupuesto que se les asigna anualmente dentro del Presupuesto de Ingresos y de Egresos del Estado se distribuye entre las mismas para su funcionamiento y para los programas, proyectos y acciones que atienden. 2.- De las entidades cuyos objetivos y fines no se relacionan con cultura. Dentro del área del Sector de Gobierno se encuentran dependencias cuyo objetivo y fines son otros, pero que tienen departamentos o programas, proyectos y acciones, que se dedican a aspectos de cultura, entre estas se pueden incluir a las que se dedican a asuntos económicos, tributarios y fiscales, y las que promueven expresiones culturales o la salvaguardia de las mismas. 2.1. De las entidades que se dedican al aspecto económico, fiscal y tributario. Entre las dependencias que se dedican a lo económico, fiscal y tributario se encuentran el Ministerio de Finanzas Públicas que es quien traslada las partidas dedicadas al sector cultura, el Ministerio de Economía que tiene programas de microcrédito que por su medio otorgan a emprendimientos culturales y a empresas culturales. También se incluye a la Superintendencia de Administración Tributaria que es quién otorga y supervisa los beneficios fiscales contenidas en las leyes de la materia. En esta parte se puede incluir al Congreso de la República que aprueba cada año el Presupuesto General de Ingresos y Egresos del Estado, como consecuencia fija las partidas que tendrá cada dependencia de gobierno y otras que se incluyen en dicho presupuesto. 2.3 .De las entidades que tienen dependencias, programas, proyectos y acciones dedicadas a la salvaguardia de expresiones culturales. Entre estas entidades se encuentran entes como el Instituto Guatemalteco de Turismo, que se dedica a promocionar y apoyar expresiones culturales, los Ministerios de Gobernación, de Relaciones Exteriores, de Economía y de Comunicaciones, que tienen dependencias específicas como la Tipografía Nacional, el diario de Centroamérica, la radio Nacional TGW, para el desarrollo de la Política Cultural en el Marco de la Política Exterior y el Registro de la Propiedad Intelectual con su Departamento de Derecho de Autor y Derechos Conexos. Se incluye en este apartado entidades como el Consejo Nacional de Áreas Protegidas CONAP, que administra con el Ministerio de Cultura, sitios arqueológicos que se encuentran en áreas protegidas, el Organismo Judicial que tiene tribunales que juzgan y dictan sentencias por delitos contra la propiedad intelectual y contra el patrimonio cultural. El Ministerio Público que tiene las Fiscalías para los delitos indicados anteriormente. Se incluye además al Fondo de Desarrollo Indígena (FODIGUA), que tiene entre sus programas apoyos para las expresiones culturales de los pueblos indígenas. En este apartado se incluye además a las municipalidades que tienen en algunos casos casas de cultura, grupos de arte, apoyos a fiestas tradicionales, bibliotecas, escuelas de arte y otros programas que le permiten su autonomía y el Código municipal. Es importante mencionar que algunos Ministerios del Organismo Ejecutivo han creado dependencias para el respeto y aplicación de los Derechos Culturales de las personas y de los pueblos indígenas, o acciones específicas como mantener conjuntos musicales para sus actividades, principalmente con marimbas.

\section{Del sector no gubernamental}

Se incluye en este apartado a las personas individuales, que son hacedoras o portadoras de expresiones culturales, y a personas individuales o jurídicas que se dedican o tienen proyectos, programas o acciones dedicadas a un área de la salvaguardia del patrimonio cultural. El sector no gubernamental es fundamental para la cultura guatemalteca, pues con o sin apoyo del sector de gobierno, desarrolla un trabajo muy importante para la salvaguardia del patrimonio cultural, sea de manera voluntaria, gratuita u onerosa, en forma individual o grupal sin personalidad jurídica o por medio de personas jurídicas en la forma de asociaciones, fundaciones o empresas comerciales.

En lo individual encontramos a los artistas y a los hacedores y portadores de expresiones culturales, que sin apoyo oficial o con poco apoyan realizan su arte y sus expresiones. En lo grupal encontramos por ejemplo hermandades religiosas y cofradías, cuyo trabajo se visibiliza en las fiestas patronales y en el ciclo cultural de la cuaresma. El sector no gubernamental es fundamental en la salvaguardia del patrimonio cultural, principalmente en el inmaterial e intangible que es identitario: de etnia, pueblo o región, pues fortalece la autoestima, el orgullo, la cohesión social y el sentido de pertenencia.

Gran parte de la salvaguardia del Patrimonio Cultural, en un porcentaje alto, incuantificable, se mantiene gracias al esfuerzo y dedicación del sector no gubernamental. Los artistas con o sin apoyos o estímulos desarrollan sus expresiones artísticas, igual sucede con quienes organizan festivales, ferias, eventos, con fiestas populares y patronales. Existen asociaciones culturales, casas de cultura, y fundaciones, cuyo objetivo es el desarrollo de expresiones de cultura o de salvaguardia de patrimonios culturales específicos. La Semana Santa, el evento cultural colectivo más importante de Guatemala, se desarrolla gracias a cofradías, hermandades 
y personas que elaboran por ejemplo alfombras de distintos productos para el paso de los cortejos procesionales. Lo mismo sucede con las artesanías y con la gastronomía, para no citar más ejemplos. Afortunadamente existen normas legales que les permiten su desarrollo, organización y funcionamiento, así como beneficios fiscales cuando corresponden.

\section{Del Ministerio de Cultura}

El Ministerio de Cultura y Deportes nació a la vida jurídica durante el Gobierno de facto del General Humberto Mejía Víctores, mediante el Decreto Ley 25-86, de fecha diez de enero de 1986, que contenía una ley para el Organismo Ejecutivo. El 14 de enero del año 2000 asumió como Ministra de Gultura y Deportes la licenciada Otilia Lux de Cotí. Una de las primeras acciones de su administración fue la de celebrar un Congreso sobre Lineamientos de Políticas Culturales y Deportivas que se realizó en La Antigua Guatemala del 4 al 11 de abril de 2000. Fueron alrededor de 600 personas las que participaron en las ocho mesas temáticas de trabajo. Más de 200 instituciones estuvieron representadas, entre ellas organizaciones mayas, garífunas y xincas, así como corporaciones municipales, organismos gubernamentales, asociaciones, fundaciones, universidades y centros de investigación.

Como consecuencia de la Declaración Mundial sobre las Políticas Culturales que tuvo lugar en México el 6 de agosto de 1982, conocida como la Declaración de México sobre las Políticas Culturales, así como de la declaración de la Conferencia Intergubernamental Sobre Políticas Culturales para el Desarrollo que se llevó a cabo en Estocolmo en 1998, y por las convenciones, recomendaciones y declaraciones de organismos internacionales como la UNESCO, de los que Guatemala es Estado-Parte, para responder a las demandas de los guatemaltecos, se crearon, en la forma que se explicó con anterioridad, las Políticas Nacionales de Cultura y Deportes. Políticas que parten de la aceptación de los Acuerdos de Paz, principalmente el de Identidad y Derecho de los Pueblos Indígenas, y de instrumentos legales nacionales, como la Constitución Política de la República en sus artículos del 55 al 66, e internacionales ratificados con los procedimientos respectivos, sobre nuestro rico y variado patrimonio cultural, así como del reconocimiento de los derechos culturales de todos los guatemaltecos y de la obligación que tiene el Estado de salvaguardar el patrimonio cultural, promover la cultura en todas sus manifestaciones y de apoyar a los artistas, a hacedores y portadores de expresiones culturales tradicionales. Las Políticas Culturales, en el año 2015, mediante un proceso participativo y similar al que se llevó cuando fueron creadas se modificaron y actualizaron, para responder a los requerimientos del sector cultura, sobre todo los relacionados con los derechos culturales de los pueblos indígenas, de los que la Dirección de Desarrollo de las Culturas dedica gran parte de su trabajo y objetivos, y por los compromisos y obligaciones adquiridas por la ratificación de instrumentos internacionales emitidos por organismos de los que Guatemala es Estado Parte.

Para responder también a nuestra realidad, como nación multicultural, plurilingüe y multiétnica, y a las políticas antes enunciadas, se elaboró un Plan Nacional de Desarrollo Cultural a Largo Plazo. En dicho Plan se hace una propuesta de desarrollo humano sostenible para Guatemala desde la óptica de la cultura, en la que el Ministerio de Cultura y Deportes es el articulador, con un ideario y una visión de gestor del desarrollo cultural. Dentro de su marco conceptual, como pilares de la propuesta, se establecen: a) La diversidad cultural como fuente de riqueza social. b) Un desarrollo social sostenible c) Un desarrollo integral sostenible. d) Una interculturalidad con equidad. e) Una efectiva descentralización y desconcentración, f) el fortalecimiento del poder local con gobernabilidad y legitimidad, g) La participación ciudadana, h) La construcción de redes, i) la construcción de ciudadanías. j) El tejido social como instrumento de desarrollo. En la metodología propuesta se explica cómo se debe abordar la dimensión cultural, sus escenarios de aplicación (intrainstitucional, interinstitucional, sociedad y nación, e internacional). En el Plan se desarrollan los ámbitos jurídicos, de ciudadanía, del estado, del pensamiento, del patrimonio, del tiempo libre, de la comunicación y de la economía.

El Ministerio de Cultura y Deportes cuenta con dependencias que se encargan cada una de un área específica de la cultura. Son casi tres mil sus trabajadores.

\section{Del Aporte para la Descentralización Cultural}

\section{(ADESCA)}

Esta entidad, creada en 1996 por una ley emitida por el Congreso de la República, realiza un trabajo extraordinario de apoyo económico a diversos sectores de la cultura, pero lo más importante es que el ochenta por ciento de los fondos que otorga cada año, a las personas o entidades que previos procesos de solicitud son favorecidas, se ejecutan en proyectos que se realizan fuera de la ciudad de Guatemala, y con sectores que tradicionalmente han sido desatendidos, logrando con ello un movimiento cultural, que puede ser medido y cuantificado por los resultados obtenidos desde 1998; 
año en el que comenzó a trabajar. ADESCA ha llegado a comunidades remotas y a grupos de personas indígenas y campesinas, que mantienen sus expresiones culturales tradicionales y conocimientos ancestrales, pero también a quienes rescatan y practican diversas expresiones artísticas, entre ellas musicales, como las del periodo barroco y contemporáneas como el Jazz y el rock. El apoyo a la creación de escuelas de marimba, la adquisición de ese instrumento y a las danzas tradicionales ha sido uno de sus grandes éxitos. Por su estructura administrativa parece una entidad de la sociedad civil, por lo que tiene mucha credibilidad en el medio cultural. Su presupuesto de funcionamiento y de inversión lo recibe del Ministerio de Cultura y Deportes.

Del Consejo de Protección de La Antigua Guatemala

Esta entidad, descentralizada, es la encargada de todo lo relacionado con la restauración y conservación de La Antigua Guatemala, ya que esta ciudad de la época de la colonia española fue declarada en 1979 como patrimonio cultural de la humanidad. El Congreso de la República le asigna su presupuesto de funcionamiento, el que le es traslado por medio del Ministerio de Cultura y Deportes.

\section{De la Academia de Lenguas Mayas}

Esta academia fue creada por una Ley del Congreso de la Republica como entidad descentralizada, y recibe su presupuesto de funcionamiento directamente del Ministerio de Finanzas. Tiene una estructura en su dirección a cargo de personas de origen maya, que son electas por los procedimientos establecidos, y su fin principal es la salvaguardia de los idiomas mayas, por lo que tienen proyectos y programas específicos. Existe como un instrumento útil para su trabajo una ley de oficialización de los idiomas de Guatemala, que establece que en cada región lingüística del país, que son 24 , se hablará para eventos y actividades públicas, indistintamente, el idioma oficial, el español, y el idioma de la región. Está establecido también qué en los primeros años de la educación formal, para quienes son hablantes de un idioma maya, está se dará en el idioma de la región, y para quienes no lo son, se darán enseñanzas en el idioma local.

\section{Del Fondo Indígena (FODIGUA)}

Este fondo, creado por Acuerdo Gubernativo, es decir por la Presidencia de la República, tiene como fin apoyar con estímulos financieros proyectos y programas para perso- nas indígenas y a entidades formadas por estos, por lo que apoya, ocasionalmente, algunos relacionados con expresiones culturales y de salvaguardia de patrimonios culturales.

\section{La importancia de crear una política de fomento para los emprendimientos, las empresas y las industrias culturales o creativas}

La cultura es un motor del desarrollo integral, de ahí que partiendo de la definición de cultura propuesta por UNESCO a sus Estados Parte, entre ellos Guatemala, que dice que «es el conjunto de rasgos distintivos, materiales, espirituales, intelectuales, y afectivos de una sociedad o grupo social, que comprende, además de las artes y las letras, los estilos de vida, las formas de convivencia, los sistemas de valores, las tradiciones y creencias» el Ministerio de Cultura y Deportes, como ente rector de la cultura en Guatemala, debe desarrollar su trabajo, cumpliendo con sus objetivos, es por ello que debe crear una política específica y de Estado, que fortalezca el trabajo que ya se hace, para que fomente a los emprendimientos, las empresas y las industrias culturales o creativas, para que este sector sea parte de las prioridades de gobierno, en el eje de fomento a las micro, pequeñas y medianas empresas, como parte de la Política Nacional de Emprendimientos «Guatemala Emprende», aprobada mediante el Acuerdo Gubernativo 150-2015, formulada por el Ministerio de Economía.

En atención a esta política, pero con el convencimiento que la cultura es un motor del desarrollo integral, en el Viceministerio de Cultura, a partir del año 2016, cuando tuve el honor de asumir dicha cartera, priorizamos el tema de Economía y Cultura, por lo que decidimos continuar con la construcción de la Cuenta Satélite de Cultura, del Sistema de información cultural, de la creación e implementación de políticas culturales municipales con los municipios que nos lo solicitaron, un convenio de cooperación con el Ministerio de Economía para el tema de emprendimientos culturales, estudios sobre el tema de dichos emprendimientos culturales, por medio de consultorías y la publicación de los mismos, así como en la consolidación de un programa para fomentar los emprendimientos culturales y las industrias creativas; temas en los que avanzamos lo suficiente hasta mi salida del cargo en enero del año en curso.

Un aspecto que para esta política y para otros temas relacionados con cultura, es el de resolver el tema de la propiedad intelectual de las expresiones culturales tradicionales y los conocimientos ancestrales por los reclamos de los 
pueblos indígenas. Esto se debe a la apropiación indebida de estos patrimonios, que pertenecen a colectividades o comunidades, por parte de terceros, quienes incluso los inscriben a sus nombres en los Registros de Propiedad Intelectual o Industrial por los procedimientos de marcas, patentes o inventos en distintos países, incluso en algunos donde no se han generado esos saberes.

Guatemala debe emitir una ley, propia, de reconocimiento de la propiedad intelectual de las expresiones culturales tradicionales y de los conocimientos tradicionales que sea coincidente con la o con las convenciones que se emitan en la OMPI, cuando termine un proceso de análisis, consulta, emisión, que ya tiene varios años de haberse iniciado en dicho organismo, ya que estas al ratificarse se convertirán en leyes del país. Estas normativas beneficiarán a los hacedores y portadores de expresiones culturales tradicionales colectivas, no solo desde el punto de vista económico sino también en cuanto al fortalecimiento de las identidades de las personas que pertenecen a uno de los cuatro pueblos que forman Guatemala. Estas leyes deben ser equilibradas y justas para que no causen enfrentamientos por titularidad, con procedimientos de registro, fáciles y accesibles. En Latinoamérica son Perú, Panamá y Brasil quienes ya tienen legislaciones sobre el tema, aunque la tendencia es a que se mejoren.

\section{De la Política Cultural Exterior}

Uno de los aspectos novedosos en cuanto al sector oficial de la cultura, es la creación en el año 2012 de la Política Cultural en el Marco de la Política Exterior, que de manera incipiente se está implementando en el país, por medio del Ministerio de Relaciones Exteriores, con el apoyo del Mi- nisterio de Cultura y Deportes, y de otras entidades como el INGUAT. Esta política es coincidente con la Política Cultural de Integración Centroamericana y con la Carta Cultural Iberoamericana, que son instrumentos regionales que están generando un desarrollo cultural centroamericano e iberoamericano, coordinados por las instancias respectivas como la CEGG/SICA y la Secretaria Iberoamericana ( SEGIB). Esta política tiene varios objetivos, pero principalmente para generar un efectiva diplomacia cultural que equilibre con la imagen negativa que del país se tiene en el extranjero y demuestre el rico y variado patrimonio cultural, entre ellos el artístico, así como una activa y productiva participación en organismos internacionales de los que Guatemala es Estado Parte, entre ellos la UNESCO, GERLALC, CECG, SEGIB, OEI, OMPI. Entre otros.

\section{Conclusiones}

Como se puede deducir el sector de gobierno, a pesar de sus limitaciones, trabaja para el apoyo a las expresiones de cultura y la salvaguardia del patrimonio cultural, aunque es mucho lo que falta por hacer, sobre todo lo que se refiere a apoyos económicos y en otras esferas, necesarias, de estímulo, protección, rescate y difusión. Sin embargo, gracias al trabajo que realiza, que se complementa con el del sector no oficial, es por lo que en Guatemala existe un intenso movimiento cultural, entre ellos el artístico, que permitirá que se consolide como un motor del desarrollo integral, para construir una sociedad con justicia social, que permita a todos y todas acceder a una vida digna y decorosa, para superar así los males contemporáneos y los ancestrales que el país arrastra, que lo han convertido en uno de los países más atrasado de América. 